\title{
Intrahepatisches Sarkom der follikulär-dendritischen Zellen
}

\author{
Fritzsche, F R ; Bode, B ; Thies, S ; Donati, O F ; Schiesser, M ; Clavien, P A ; Jochum, W
}

\begin{abstract}
Wir berichten über ein primär intrahepatisches Sarkom der follikulär-dendritischen Zellen, das bei einer 76-jährigen Patientin auf dem Boden eines Morbus Castleman vom hyalin-vaskulären Typ auftrat. Wir diskutieren die klinisch-pathologischen Befunde, die Pathogenese und das differenzialdiagnostische Vorgehen bei diesem seltenen Tumortyp = We report an intrahepatic sarcoma of the follicular dendritic cells in a 76-year-old woman with a medical history of a hyaline-vascular type of Castleman's disease. We discuss the clinico-pathological findings, the pathogenesis and the differential diagnosis of this rare tumour entity.
\end{abstract}

DOI: https://doi.org/10.1007/s00292-009-1163-y

Other titles: Intrahepatic sarcoma of the follicular dendritic cells

Posted at the Zurich Open Repository and Archive, University of Zurich

ZORA URL: https://doi.org/10.5167/uzh-20913

Journal Article

Published Version

Originally published at:

Fritzsche, F R; Bode, B; Thies, S; Donati, O F; Schiesser, M; Clavien, P A; Jochum, W (2010). Intrahepatisches Sarkom der follikulär-dendritischen Zellen. Der Pathologe, 31(2):153-156.

DOI: https://doi.org/10.1007/s00292-009-1163-y 
Pathologe 2010 $\cdot 31: 153-156$ DOI 10.1007/s00292-009-1163-y

Online publiziert: 1. Juli 2009

c) Springer Medizin Verlag 2009

\author{
F.R. Fritzsche ${ }^{1} \cdot$ B. Bode ${ }^{1} \cdot$ S. Thies ${ }^{1} \cdot$ O.F. Donati ${ }^{2} \cdot$ M. Schiesser ${ }^{3} \cdot$ P.A. Clavien ${ }^{3}$. \\ W. Jochum ${ }^{4}$ \\ ${ }^{1}$ Institut für Klinische Pathologie, UniversitätsSpital Zürich, Schweiz \\ ${ }^{2}$ Institut für Diagnostische Radiologie, Zürich, Schweiz \\ ${ }^{3}$ Klinik für Viszeral- und Transplantationschirurgie, UniversitätsSpital Zürich, Schweiz \\ ${ }^{4}$ Institut für Pathologie, Kantonsspital St. Gallen, Schweiz
}

\section{Intrahepatisches Sarkom der follikulär- dendritischen Zellen}

liert und in kleinen Aggregaten liegende spindelige Zellen mit vergrößerten pleomorphen Kernen, vergröberter Chromatinstruktur und teils prominenten Nukleolen. Eine Panzytokeratin-Immunzytochemie fiel negativ aus.

Die Stanzbiopsie zeigte eine ausgedehnte Sklerosezone, in der nestartig kleine Gruppen atypischer Zellen mit Kernpleomorphie und prominenten Nukleolen darstellbar waren ( $\bullet$ Abb. 4 a). Die atypischen Zellen waren immunhisto- chemisch negativ für Panzytokeratin, Melan $\mathrm{A}, \mathrm{CD}_{31}, \mathrm{CD}_{34}, \mathrm{HHV} 8, \mathrm{CD}_{45}, \mathrm{CD}_{3}$, $\mathrm{CD}_{15}, \mathrm{CD}_{30}, \mathrm{CD}_{23}$ und ALK1. Einzelne atypische Zellen zeigten eine Positivität für $\mathrm{CD}_{21}(\boldsymbol{\bullet} \mathbf{a b b} .4 \mathbf{b})$. Die Proliferationsrate (Ki67-Index) betrug 20-30\%.

Im Leberteilresektat $(1314 \mathrm{~g})$ fanden sich 2 derbe weißliche, bis $13 \mathrm{~cm}$ große Tumoren in nichtzirrhotischem Leberparenchym (- Abb. 5 a). Histologisch entsprachen die Tumoren großen Sklerosezonen mit darin eingelagerten Herden von worden. Zusätzlich lag bei der histologischen Untersuchung der Lymphknoten eine herdförmige Vermehrung der follikulär-dendritischen Zellen (FDC) vor, die als nichtneoplastische FDC-Hyperplasie beurteilt wurde ( $\mathrm{Abb} .1 \mathrm{a}, \mathbf{b})$. Zur Abklärung der Leberherde wurden zunächst eine Feinnadelpunktion und nachfolgend bei Verdacht auf einen malignen Tumor eine Stanzbiopsie durchgeführt.

\section{Klinische Befunde}

Die Magnetresonanztomographie (MRT) der Leber zeigte in den Segmenten V und VIII 2 intrahepatische, in der T2-gewichteten Sequenz hyperintense Raumforderungen von maximal 10,4 cm Durchmesser (- Abb. 2). Das weitere Staging verlief negativ.

\section{Pathologische Diagnostik}

Das Feinnadelpunktat (• Abb. 3 a, b) zeigte neben normalen Hepatozyten iso-

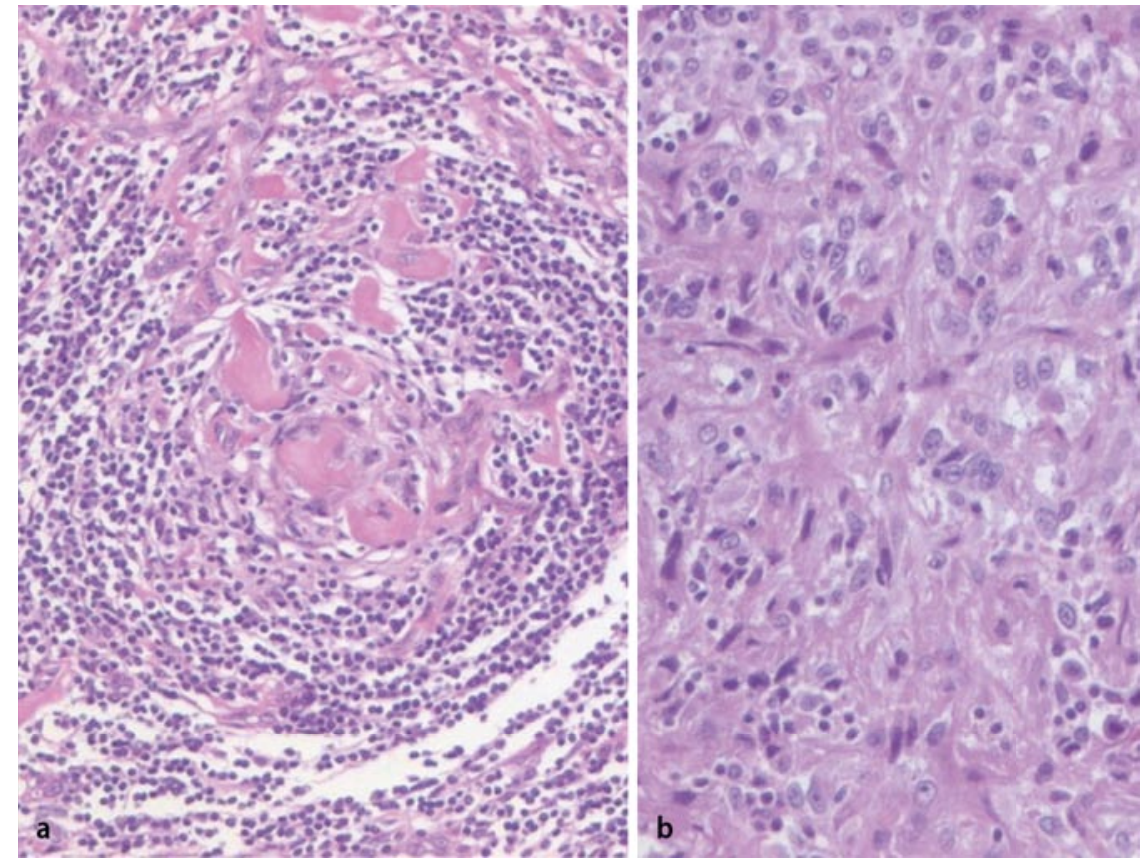

Abb. $1 \Delta$ a, b M. Castleman mit herdförmiger Vermehrung der follikulär-dendritischen Zellen in den Lymphknoten 


\section{Fallberichte}
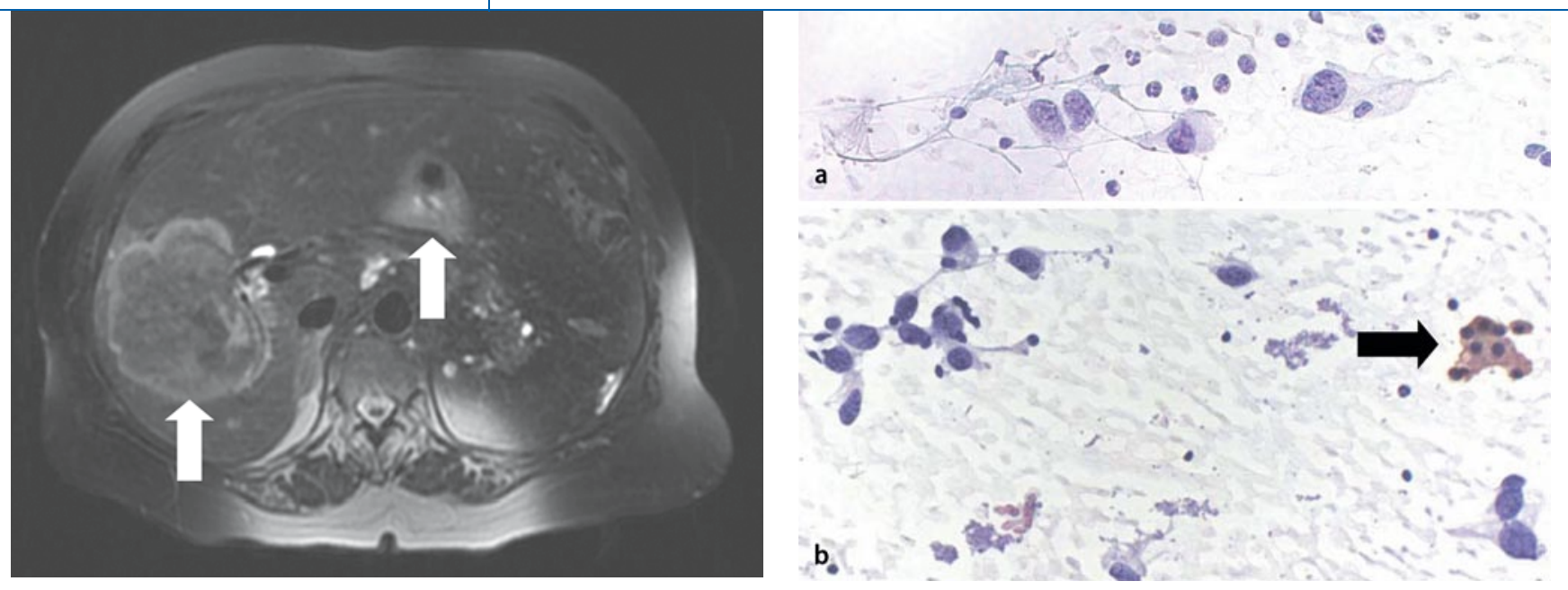

Abb. $2 \triangle$ Magnetresonanztomographie der Leber mit Tumorherden (Pfeile)

Abb. $3 \Delta$ a, b Zytologie des FDC-Sarkoms und normale Hepatozyten (Pfeil)

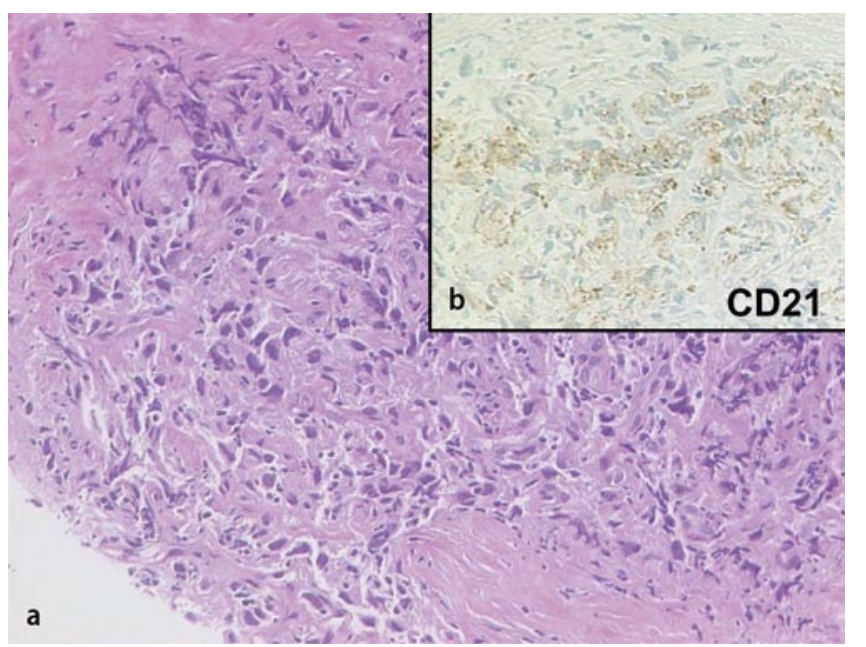

Abb. $4<$ Stanzbiopsie. a Nestartige Gruppen atypischer Zellen mit Kernpleomorphie und prominenten $\mathrm{Nu}$ kleolen. b Positivität für CD21
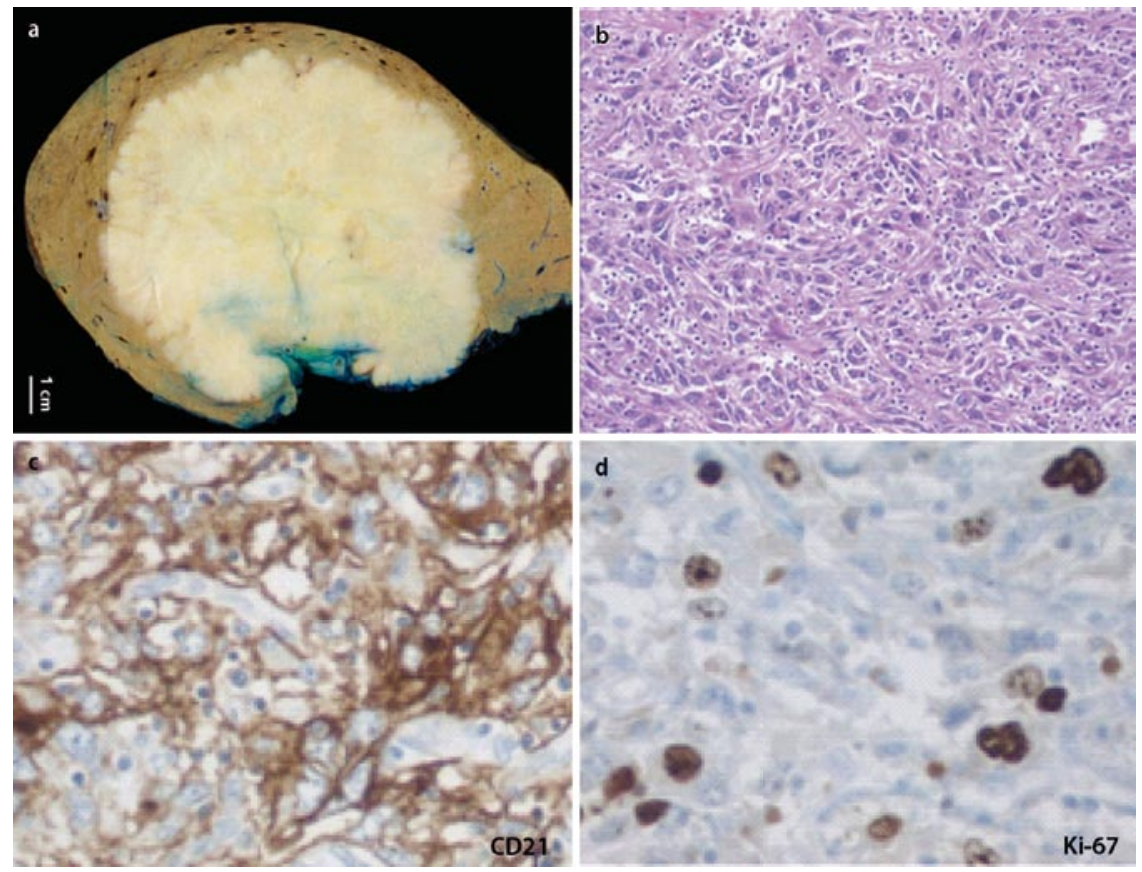

Abb. $5 \Delta$ Makroskopie (a) und Exzisionshistologie (b-d) des FDC-Sarkoms mit umgebender Fibrose in nichtzirrhotischer Leber spindelzelligen und epitheloiden Tumorzellen, die teils eine deutliche Kernpleomorphie und atypischen Mitosen zeigten (- Abb. 5 b). Herdförmig war eine Gefäßinvasion nachweisbar. Immunhistologisch waren die Tumorzellen negativ für HepPar1, CK7, CK19, EMA, CEA, CD23, GLUT1, Claudin-1, S10o, CD68 und D240 bei Nachweis einer deutlichen CD21Expression (- Abb. 5 c). Die Proliferationsrate betrug 10-20\% (• Abb. 5 d). Eine EBER-in-situ-Hybridisierung fiel negativ aus. Zusammenfassend wurde ein FDC-Sarkom mit Gefäßinvasion diagnostiziert.

\section{Therapie und Verlauf}

Nach komplikationslosem postoperativem Verlauf wurde die Patientin nach Hause entlassen. Sieben Wochen darauf erfolgte eine notfallmäßige Hospitalisierung im septischen Schock bei Perforation eines Jejunumdivertikels. Nach erneuter Operation und kompliziertem Verlauf mit multiplen Abdominaleingriffen und persistierender Sepsis verstarb die Patientin an einer in der Autopsie gesicherten akuten abszedierenden Pankreatitis. Weitere Anteile des FDC-Sarkoms oder des M. Castleman wurden nicht gefunden.

\section{Diskussion}

Extranodale FDC-Sarkome sind sehr selten und wurden in verschiedenen Lokalisationen (Tonsillen, Gastrointestinaltrakt, Weichgewebe, Mamma, Nasopharynx 
und Leber) beobachtet [5]. Hier berichten wir über die klinisch-pathologischen Befunde eines primär intrahepatischen FDC-Sarkoms, von dem bisher nur 7 Fälle in der Literatur beschrieben wurden [3, $8,9]$.

Die im vorliegenden Fall beobachteten klinisch-pathologischen Befunde entsprechen weitgehend denen der beschriebenen FDC-Sarkome der Leber. Das Erkrankungsalter der Patienten lag zwischen 35 und 82 Jahren. Es waren vor allem Frauen betroffen. Die Tumoren waren häufiger in den rechtsseitigen Lebersegmenten lokalisiert und hatten bei Diagnosestellung eine Größe zwischen $9,5 \mathrm{~cm}$ und $20 \mathrm{~cm}$. Makroskopisch lag in der Regel ein umschriebenes Tumorwachstum vor. Histologisch zeigte der Tumor wie in der Mehrzahl der anderen hepatischen FDC-Sarkome ein kohäsives Wachstum der Tumorzellen. Während in unserem Fall die Tumorzellverbände in einem sklerosierten Stroma mit einem geringen lymphozytären Entzündungsinfiltrat lagen, wurden auch Tumoren mit einem dichten lymphoplasmazellulären Infiltrat (inflammatorisch-pseudotumorähnlich) und vereinzelt gelagerten Tumorzellen beschrieben. Zytologisch waren die Tumorzellen spindelzellig bis ovoid und zeigten teilweise nukleäre Pseudoeinschlüsse und Kernkerben.

Konventionell-morphologisch wurde an der Leberbiopsie die Manifestation eines Karzinoms, Sarkoms, Melanoms oder Lymphoms in Betracht gezogen. Aus der breiten Differenzialdiagnose ergibt sich, dass eine rein konventionelle $\mathrm{Di}$ agnosestellung eines FDC-Sarkoms nicht mit genügender Sicherheit möglich ist und molekulare Zusatzuntersuchungen durchgeführt werden sollten. FDC und deren Tumoren sind meist immunhistochemisch positiv für $\mathrm{CD}_{21}, \mathrm{CD}_{23}, \mathrm{CD}_{35}$, KiM4p oder CNA.42. Bei genügend Material ist nach unserer Erfahrung mit einem weiteren Fall eines extranodalen FDC-Sarkoms die Diagnosestellung auch immunzytologisch möglich. Eine starke Expression von Clusterin, einem allerdings nicht FDC-spezifischen Glykoprotein, soll typisch für das FDC-Sarkom sein und könnte in sehr seltenen CD21/23/35negativen Fällen hilfreich sein [7].
Der Entstehungsmechanismus der FDC-Sarkome ist unbekannt. FDC gelten als die Ausgangszellen dieses Tumortyps. Sie kommen als ortsgebundene antigenpräsentierende Zellen ubiquitär in nodalen und extranodalen Lymphfollikeln vor.

Der sequenzielle Übergang von FDCHyperplasie in ein FDC-Sarkom wurde vermutet $[1,9]$. Zu dieser Annahme passt unsere Beobachtung, dass bereits bei der Diagnose des M. Castleman eine Vermehrung der FDC in den Lymphknoten vorlag. Vor allem die inflammatorisch-pseudotumorähnliche Variante des FDC-Sarkoms zeigte in den meisten berichteten Fällen eine Assoziation mit einer EpsteinBarr-Virusinfektion $[1,9]$. Wir fanden keine Hinweise auf eine solche Infektion.

In einigen Fällen, wie in dem hier beschriebenen, entstand das FDC-Sarkom auf dem Boden eines M. Castleman vom hyalin-vaskulären Typ, so dass diese Erkrankung als Prädisposition für ein FDC-Sarkom angesehen werden kann $[1,4,6,9]$.

\section{Fazit für die Praxis}

Intrahepatische Sarkome der follikulärdendritischen Zellen sind sehr selten.

Dennoch empfiehlt es sich, bei Vorliegen eines spindelzelligen Tumors auch ein FDC-Sarkom in Betracht zu ziehen und immunhistologische Untersuchungen in diese Richtung (CD21, CD23, CD35, Clusterin) durchzuführen. Eine Assoziation mit dem M. Castleman vom hyalin-vaskulären Typ ist etabliert, so dass ein FDCSarkom v. a. im Kontext eines M. Castleman differenzialdiagnostisch erwogen werden muss.

\section{Korrespondenzadresse}

\section{Dr. F.R. Fritzsche}

Institut für Klinische Pathologie, UniversitätsSpital Zürich

Schmelzbergstr. 12, 8091 Zürich, Schweiz

Florian.Fritzsche@usz.ch

Interessenkonflikt. Der korrespondierende Autor gibt an, dass kein Interessenkonflikt besteht.
Pathologe 2010 · 31:153-156

DOI 10.1007/s00292-009-1163-y

(c) Springer Medizin Verlag 2009

F.R. Fritzsche - B. Bode - S. Thies - O.F. Donati $\cdot$ M. Schiesser · P.A. Clavien · W. Jochum Intrahepatisches Sarkom der follikulär-dendritischen Zellen

Zusammenfassung

Wir berichten über ein primär intrahepatisches Sarkom der follikulär-dendritischen Zellen, das bei einer 76-jährigen Patientin auf dem Boden eines Morbus Castleman vom hyalin-vaskulären Typ auftrat. Wir diskutieren die klinisch-pathologischen Befunde, die Pathogenese und das differenzialdiagnostische Vorgehen bei diesem seltenen Tumortyp.

Schlüsselwörter

FDC-Sarkom · Morbus Castleman · Leber .

Zytologie $\cdot$ Autopsie

\section{Intrahepatic sarcoma of the} follicular dendritic cells

\section{Abstract}

We report an intrahepatic sarcoma of the follicular dendritic cells in a 76-year-old woman with a medical history of a hyaline-vascular type of Castleman's disease. We discuss the clinico-pathological findings, the pathogenesis and the differential diagnosis of this rare tumour entity.

Keywords

FDC sarcoma - Castleman disease - Liver . Cytology · Autopsy 


\section{Literatur}

1. Chan AC, Chan KW, Chan JK et al (2001) Development of follicular dendritic cell sarcoma in hyaline-vascular Castleman's disease of the nasopharynx: tracing its evolution by sequential biopsies. Histopathology 38:510-518

2. Gaffney RL, Feddersen RM, Bocklage TJ et al (2000) Fine needle aspiration cytology of follicular dendritic cell sarcoma. Report of a case with cytologic detection in an extranodal site. Acta Cytol 44:809814

3. Granados R, Aramburu JA, Rodriguez JM et al (2008) Cytopathology of a primary follicular dendritic cell sarcoma of the liver of the inflammatory pseudotumor-like type. Diagn Cytopathol 36:4246

4. Kazakov DV, Morrisson C, Plaza JA et al (2005) Sarcoma arising in hyaline-vascular Castleman disease of skin and subcutis. Am J Dermatopathol 27:327-332

5. Shia J, Chen W, Tang LH et al (2006) Extranodal follicular dendritic cell sarcoma: clinical, pathologic, and histogenetic characteristics of an underrecognized disease entity. Virchows Arch 449:148-158

6. Sun X, Chang KC, Abruzzo LV et al (2003) Epidermal growth factor receptor expression in follicular dendritic cells: a shared feature of follicular dendritic cell sarcoma and Castleman's disease. Hum Pathol 34:835-840

7. Swerdlow SH, Campo E, Harris NL et al (2008) WHO classification of tumours of haematopoetic and lymphoid tissues. IARC, Lyon

8. Torres U, Hawkins WG, Antonescu CR et al (2005) Hepatic follicular dendritic cell sarcoma without Epstein-Barr virus expression. Arch Pathol Lab Med 129:1480-1483

9. Youens KE, Waugh MS (2008) Extranodal follicular dendritic cell sarcoma. Arch Pathol Lab Med 132:1683-1687

\section{Pathologen dringend gesucht}

„Die Krankenkassen sehen keinen Ärztemangel - wir schon", so der Präsident des Bundesverbandes Deutscher Pathologen Professor Werner Schlake. Für das Fachgebiet Pathologie wurden $2008 \mathrm{im}$ Deutschen Ärzteblatt insgesamt 176 offene Stellen angeboten. Das sind circa 14\% aller Stellen (1272) überhaupt. Innerhalb dieser Stellenangebote lag der größte Bedarf mit 70\% aller Inserate im Krankenhaussektor. Im Bereich der Akutkrankenhäuser und Universitätskliniken waren 123 offene Stellen zu verzeichnen.

Die meisten Stellenangebote kamen aus Baden-Württemberg mit 35 Anzeigen, gefolgt von Bayern mit 13 und Nordrhein mit 12 Inseraten. Der Anteil der Stellenanzeigen aus dem Ausland betrug 12\%. Von den 22 ausgeschriebenen Stellen im Ausland wurde knapp die Hälfte in der Schweiz angeboten. Es folgten nennenswert noch Dänemark und Schweden mit 6 bzw. 3 Stellenangeboten. Den offenen Stellen steht eine sinkende Zahl von Pathologen gegenüber. Gab es im Jahr 2004 noch 1303 berufstätige Pathologen in Deutschland, so sind es vier Jahre später nur noch 1272. Bei den an der Klinik tätigen Pathologen war der Rückgang von $13 \%$ zwischen 2004 (646) und 2008 (561) noch deutlicher. Von den im Jahr 2008 tätigen 1272 Pathologen arbeiteten 561 Pathologen im Krankenhausbereich, 632 in der Niederlassung, 13 in Behörden und Körperschaften sowie 66 in sonstigen Bereichen.

Für Professor Schlake ist die von der Bundesärztekammer vorgenommene Auswertung der Stellenanzeigen bezüglich der einzelnen Facharztgruppen alarmierend: „Im Bereich der Tumordiagnostik werden die Untersuchungszahlen steigen und damit mehr Pathologen gebraucht, insbesondere auch weil die methodischen Anforderungen sehr viel anspruchsvoller werden."

Mit dem Einsatz molekular begründeter zielgerichteter Therapeutika hat sich ein Paradigmenwechsel in der Aufgabenstellung moderner Tumorpathologie ergeben.

„Der Pathologe ist gefordert, die Wirksamkeit einer Therapie durch Nachweis entsprechender Zielstrukturen am Gewebe vorherzusagen. Das bedeutet, er nimmt eine Schlüsselposition dafür ein, dass der richtige Patient mit dem richtigen Therapeutikum behandelt wird", umreißt Professor Schlake die Rolle der Pathologie. Vor dem Hintergrund dieser neuen Aufgaben sei der Mangel an Pathologen besonders beunruhigend.

Quelle: Bundesverband Deutscher Pathologen e. V. 